\title{
Review of tocilizumab in the treatment of rheumatoid arthritis
}

\section{Yasuaki Okuda}

Department of Internal Medicine, Center for Rheumatic Diseases, Dohgo Spa Hospital, Matsuyama-city, Ehime, Japan
Correspondence:Yasuaki Okuda Department of Internal Medicine, Center for Rheumatic Diseases, Dohgo Spa Hospital, 2 I-2 I Otsu Dohgo-Himezuka Matsuyama-city, Ehime 790-0858, Japan Tel: +8I-89-933-5 | 3 |

Fax: $+81-89-933-5137$

Email stardust@md.pikara.ne.jp

\begin{abstract}
Constitutively overproduced in proliferating synovial tissues, interleukin-6 (IL-6) is deeply involved in the pathology of rheumatoid arthritis (RA). Tocilizumab is a humanized anti-human IL-6 receptor antibody that binds to soluble and membrane-bound IL-6 receptor, and at detectable levels in blood, tocilizumab is capable of almost completely blocking the transmembrane signaling of IL-6. In clinical trials for patients with RA in Japan, tocilizumab monotherapy has shown clinical efficacy equaling that of tumor necrosis factor (TNF) inhibitor in combination with methotrexate, and in an extension study in patients who responded to tocilizumab, almost no patients showed a decrease in the efficacy of tocilizumab. Evidence obtained in a phase III study in Japan demonstrated that tocilizumab monotherapy had a significant inhibitory effect on the progression of structural joint damage compared with that of conventional disease modifying antirheumatic drugs (DMARDs). Furthermore it has been shown that tocilizumab has an excellent ability to suppress serum amyloid A levels and could therefore be an important therapeutic strategy in amyloid A amyloidosis secondary to rheumatic diseases. The safety profile of tocilizumab appears to be satisfactory. However, several serious infections were also reported, and careful monitoring is therefore important during use.
\end{abstract}

Keywords: tocilizumab, rheumatoid arthritis, interleukin-6, treatment, amyloidosis, biologics

\section{Introduction}

Rheumatoid arthritis (RA) is a chronic inflammatory disease that causes inflammation in intra-articular synovial tissue, producing pain and swelling. Persistent synovitis produces destruction of bone and cartilage, adversely affecting the activities of daily living. Extra-articular complications also often occur, thereby eliciting a worsening of the prognosis. The causes of RA are still obscure, but research using disease models has shown that proinflammatory cytokines such as tumor necrosis factor- $\alpha$ (TNF- $\alpha$ ) and interleukin (IL)-6 or IL-1 play key roles in its pathogenesis. The anti-TNF agents infliximab, etanercept, and adalimumab are presently used in the treatment of RA, and compared with methotrexate (MTX) and other conventional disease modifying antirheumatic drugs (DMARDs), not only yield greater reductions in the level of disease activity, but also have the benefit of inhibiting or partially improving the progression of structural joint damage, and are therefore aggressively used in patients with severe disease activity. For patients with disease that is resistant to TNF inhibitors, cytotoxic T lymphocyte-associated antigen-4-Ig (CTLA4-Ig) and the human $\mathrm{IgG}_{1}$ fusion protein: abatacept, and the anti-CD20 chimeric antibody: rituximab are used mainly in the US and Europe, and while they are recognized as useful, inadequate responses are still seen in no small number of cases. Meanwhile, it has been shown in clinical trials conducted to date that the humanized anti-IL 6 receptor antibody: tocilizumab has an excellent improvement effect on disease activity, an inhibitory effect on joint destruction, and therapeutic benefit for amyloid A (AA) amyloidosis. Tocilizumab has been 
filed for approval in Japan and is currently completing the global phase III clinical development program for registration in other countries. With its mechanism of action unlike that of other biologic agents and its unique characteristics, expectations are high for tocilizumab as a new therapeutic tool in the treatment of RA.

\section{Pathological role of IL-6 in RA}

Originally identified in 1982 as an antigen-nonspecific B cell differentiation factor produced by activated mononuclear cells (Yoshizaki et al 1982), IL-6 is a cytokine cloned in 1986 by Hirano et al (1986). Subsequent research has shown that IL-6 is a pleiotropic cytokine with diverse physiological actions, regulating the immune response, inflammation, bone metabolism, and hematopoiesis (Figure 1). In the joints of patients with RA, IL-6 is produced in large quantities from synovial cells and macrophages, eliciting polyclonal hypergammaglobulinemia and the emergence of autoantibodies as a result of $\mathrm{B}$ cell differentiation. Additionally, via enhanced expression of intercellular adhesion molecule-1, IL-6 promotes the infiltration of inflammatory cells into synovial tissue, and acting synergistically with TNF- $\alpha$ and IL-1, promotes angiogenesis as a result of increased production of vascular endothelial growth factor (VEGF) (Nakahara et al 2003). IL-6 also promotes increased production of metalloproteinases (Roux-Lombard et al 2001) and the activation of osteoclasts (Kotake et al 1996) is involved in the destruction of cartilage and bone. Additionally, overproduction of IL-6 leads to increases in acute phase reactants such as C-reactive protein (CRP), serum amyloid A (SAA), hypoalbuminemia, increased platelet count, and anemia, and long-term persistence of such overproduction is thought to be involved in the development of AA amyloidosis, a serious complication of RA. Recent research has also demonstrated that there are 4 subsets of helper T cells: Th1, Th2, and Th17, which produces IL-17 and other cytokines that play a key role in inflammation, and regulatory $\mathrm{T}$ cell (Treg), which is involved in negative immune response control. Th17 is induced when transforming growth factor- $\beta$ (TGF- $\beta$ ) and IL-6 are present during antigen stimulation of naïve T cells. Since the development of arthritis can be inhibited by blocking IL-17, Th17 plays a crucial role in arthritis models (Lubberts et al 2004). In contrast, Treg is only induced when TGF- $\beta$ is added to naïve T cells. In a model of arthritis induced by passive transfer of collagen-sensitized T cells, the onset of arthritis was inhibited when Treg was added simultaneously (Morgan et al 2005). IL-6 inhibits differentiation of Treg, and increases the number of Th17 cells. This demonstrates that IL-6 is an extremely important cytokine in the pathogenesis of RA, in which activated T cells

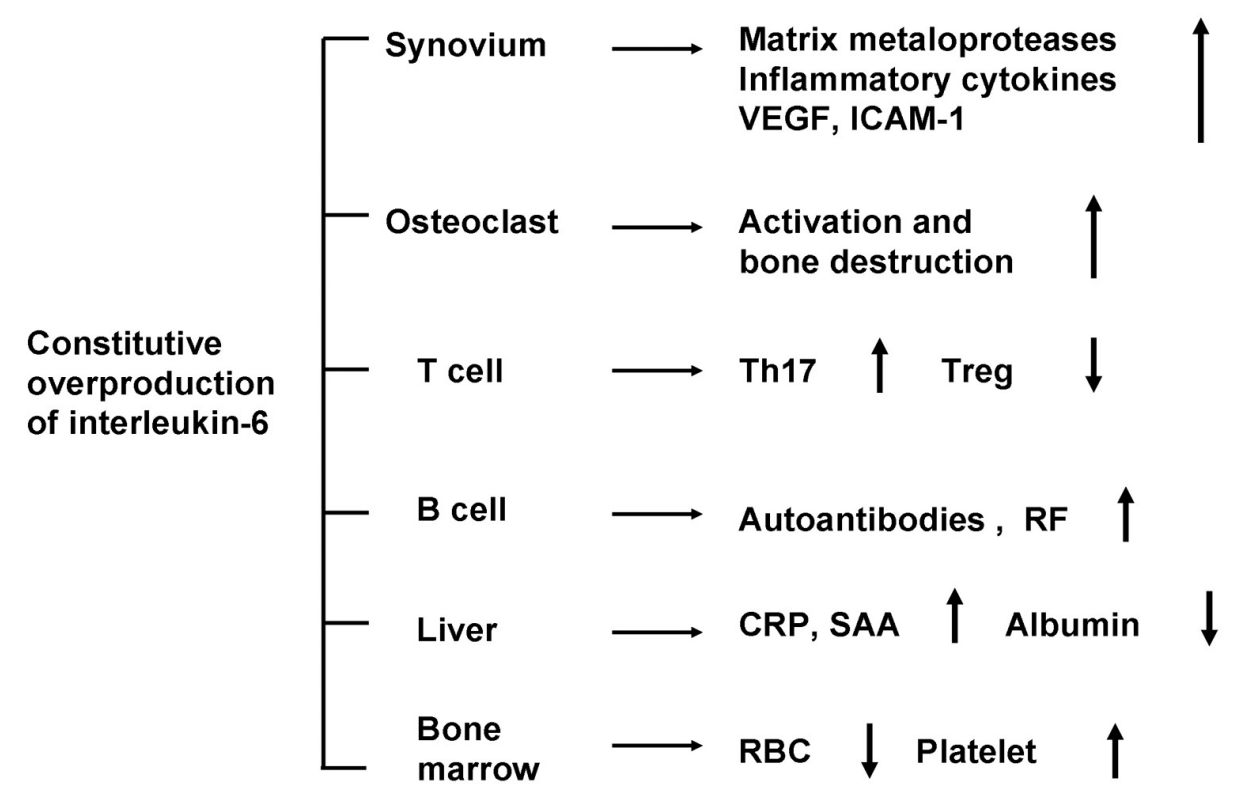

Figure I Pathological roles of interleukin-6 in rheumatoid arthritis. Constitutive overproduction of interleukin-6, a pleiotropic cytokine that regulates the immune system, inflammation, hematopoiesis, and bone metabolism, is thought to play pathologic roles in rheumatoid arthritis.

Abbreviations: VEGF, vascular endothelial growth factor; ICAM-I, intercellular adhesion molecule-I;Th I7, interleukin-I7 producing CD4+ T cell; Treg, CD4+CD25+ regulatory T cell; RF, rheumatoid factor; CRP, C-reactive protein; SAA, serum amyloid A; RBC, red blood cell. 
are involved. In contrast, even in the presence of TGF- $\beta$, TNF and IL-1 show no activity to induce Th17 or inhibit the differentiation of Treg.

\section{Mode of action of tocilizumab}

IL-6 has a unique receptor system, and while IL-6 receptor (R) specifically binds to IL-6, it does not have a direct involvement in signal transduction. IL-6 forms a complex by binding to IL- $6 \mathrm{R}$ on the cell membrane, which then combines with gp130, which also resides on the cell membrane, forming a homodimer and giving rise to intracellular signal transduction. Furthermore, IL-6R also exists as a soluble from. After forming a complex with IL-6, soluble (s) IL-6R can also combine with gp130 on the cell membrane and enables signaling (Ward et al 1994). s IL-6R is present in the blood of healthy individuals at a concentration of several tens of $\mathrm{ng} / \mathrm{mL}$, and if the cells express gp130, IL-6 signaling can take place, even in the absence of IL-6R expression on the cell membrane. Reflecting this characteristic, the effects of IL- 6 are diverse, and it is thought to have an extensive regulatory role, with involvement in the immune response, inflammation, bone metabolism, hematopoiesis, and the neuroendocrine system.

Tocilizumab is a recombinant monoclonal antibody that has been humanized by complementarity determining region (CDR) grafting of a murine anti-human IL-6R antibody onto human $\operatorname{IgG}_{1}$. Tocilizumab inhibits the induction of biological activity due to IL-6 in cells that have expressed both membrane-bound IL-6R and gp130 molecules, and also inhibits the induction of biological activity due to IL-6/IL-6R complex formation in cells that express gp130 alone. Furthermore, since it has the capacity to dissociate IL-6/IL-6R complexes that have already formed, it exhibits an extremely effective blocking action on IL-6 signal transduction (Figure 2).

\section{Clinical trials of tocilizumab for RA Phase I clinical trial (UK)}

A phase I clinical trial to confirm the safety and efficacy of tocilizumab in single-dose administration was conducted in the United Kingdom (Choy et al 2002). A single dose of tocilizumab $0.1,1,5,10 \mathrm{mg} / \mathrm{kg}$ or placebo was given

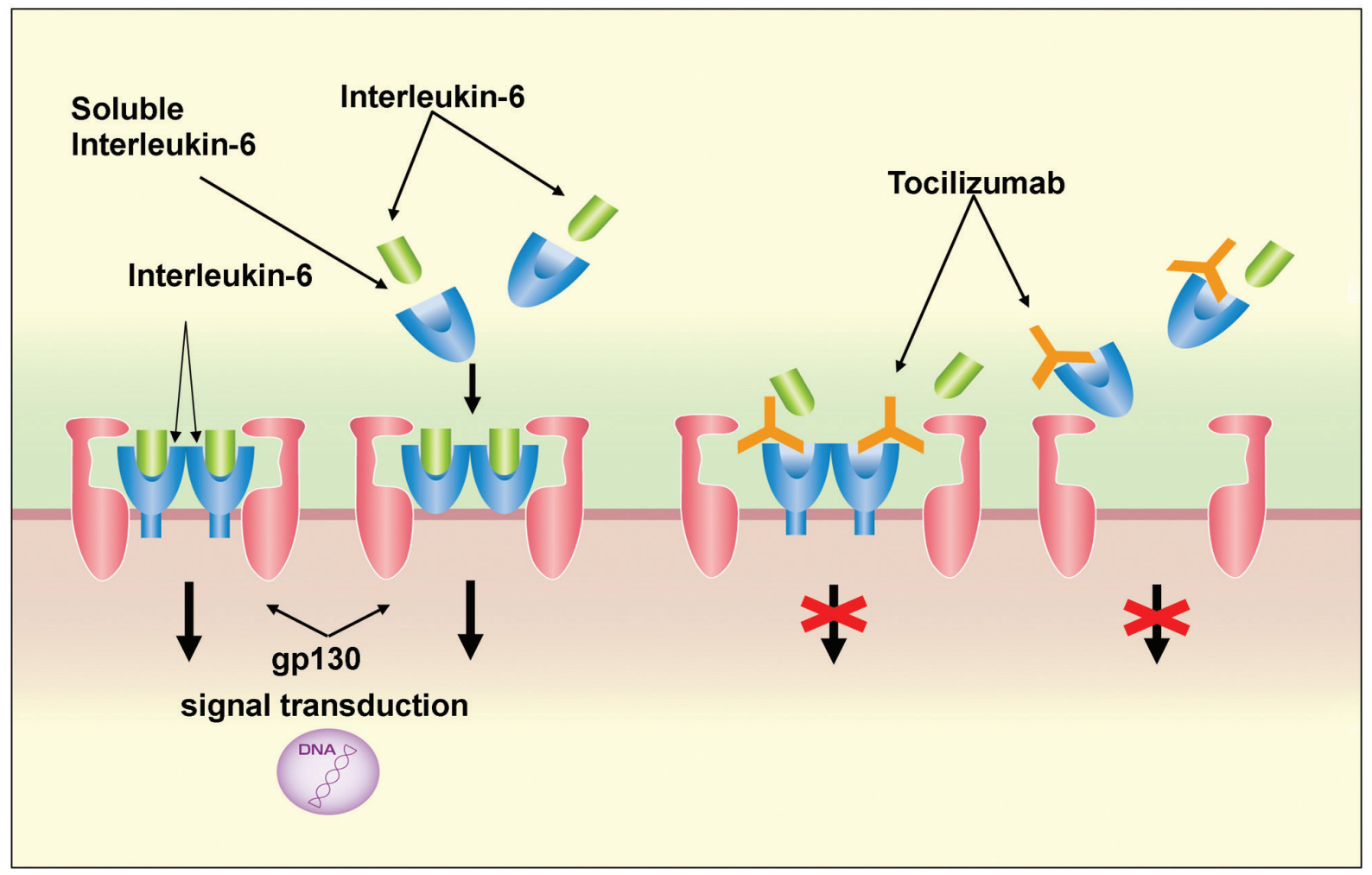

Figure 2 Signal transduction of interleukin (IL)-6 /L-6 receptor (R) complex via gp I30 and the mechanism of signal transduction blockage by tocilizumab. IL-6 signal is mediated via IL-6R on the cell surface or soluble form of IL-6R, followed by dimerization of the signal transducer gp-130, which is bound to the IL-6/L-6R complex. Tocilizumab inhibits the binding of IL-6 to IL-6R or sIL-6R. 
to a total of 45 patients with RA, and an analysis of pharmacokinetics and evaluation of RA disease activity were performed at 2 weeks post-dose. An ACR20 response, indicating 20\% improvement in disease activity according to the American College of Rheumatology (ACR) criteria, was achieved by $55.6 \%$ of patients in the $5-\mathrm{mg} / \mathrm{kg}$ group, a statistically significant difference from that in the placebo group. Furthermore, CRP levels normalized in all patients in the 5 and $10-\mathrm{mg} / \mathrm{kg}$ groups. There were no serious adverse reactions related to tocilizumab.

\section{Phase I/II clinical trials (Japan)}

A phase I/II clinical trial to investigate the pharmacokinetics and safety of tocilizumab in repeated administration was conducted in Japan (Nishimoto et al 2003). In this trial, tocilizumab 2, 4, or $8 \mathrm{mg} / \mathrm{kg}$ was administered by intravenous drip infusion every 2 weeks for a total of 3 times. Pharmacokinetics, safety, and clinical efficacy were evaluated, and if there were no safety concerns, treatment was continued and RA activity was re-evaluated at 6 months. At 4 and $8 \mathrm{mg} / \mathrm{kg}$, tocilizumab showed a tendency to accumulate in blood, and the half-life after 3 infusions of $8 \mathrm{mg} / \mathrm{kg}$ was approximately 240 hours, approaching that of human IgG. Both CRP and SAA were completely normalized in all patients in whom tocilizumab was detected in blood at the trough concentration. Based on these findings, it was confirmed that IL-6 is a cytokine that is indispensable for the production of CRP and SAA. While this was an open-label trial, the ACR20 response was $60 \%$ and the ACR50 response was $13 \%$ at 6 weeks, and with continued treatment, the ACR20 response was $86 \%$ and the ACR50 response was $33 \%$ at 6 months. MTX was not coadministered, and no neutralizing antibodies to tocilizumab were detected, confirming the advantage of a humanized antibody. In repeated administration, there was no attenuation of effects, nor were there any serious adverse events.

\section{Late phase II clinical trial (Japan)}

A late phase II clinical trial, conducted as a multicenter, double-blind, placebo-controlled trial, was started in Japan in 2001 (Nishimoto et al 2004). One hundred sixty-four RA patients with inadequate responses to one or more antirheumatic drugs or immunosuppressants were randomly assigned to 3 treatment groups: placebo, $4 \mathrm{mg} / \mathrm{kg}$, and $8 \mathrm{mg} / \mathrm{kg}$. Tocilizumab or placebo was intravenously infused every 4 weeks, a total of 3 times, and RA activity was evaluated at 12 weeks. This regimen was designed on the assumption that the blood concentration could be maintained in treatment at $8 \mathrm{mg} / \mathrm{kg}$ every 4 weeks, based on a pharmacokinetics simulation using the results of the phase I/II trial. The median number of antirheumatic drugs already used by patients before entry into the trial was 4-5, and the mean duration of disease was about 8 years, together indicating that most of these patients had progressed disease. At 12 weeks, an ACR20 response was achieved by $78 \%$ of patients in the $8-\mathrm{mg} / \mathrm{kg}$ group and $57 \%$ of patients in the $4-\mathrm{mg} / \mathrm{kg}$ group, versus a meager $11 \%$ of patients in the placebo group. Hence, improvement of the RA activity in the $8 \mathrm{mg} / \mathrm{kg}$ group was statistically significant in comparison with that in the placebo and 4-mg/kg groups $(\mathrm{p}<0.001, \mathrm{p}=0.02)$ (Figure 3). Furthermore, both the ACR50 and ACR70 responses in the 8- $\mathrm{mg} / \mathrm{kg}$ group were superior to those in the placebo group ( $\mathrm{p}<0.001, \mathrm{p}=0.002)$. Evaluation using the DAS28 (Disease Activity Score according to the European League Against Rheumatism [EULAR] improvement criteria) also showed that the response was good or moderate for $90.9 \%$ of patients in the $8-\mathrm{mg} / \mathrm{kg}$ group. The safety analysis showed that the incidences of adverse events were not dose dependent: $56 \%, 59 \%$, and $51 \%$ of the patients in placebo, $4 \mathrm{mg} / \mathrm{kg}$, and $8 \mathrm{mg} / \mathrm{kg}$ groups, respectively. The most common adverse event was upper respiratory infection (common cold), but there were no differences among the groups in its incidence. Infusion reactions were seen rarely, but these were mild and transient. Serious adverse events were reported for 3 patients receiving tocilizumab and 2 patients receiving placebo. For 1 patient in the tocilizumab group, chronic, active Epstein-Barr virus (EBV) infection was reactivated after the first infusion of $8 \mathrm{mg} / \mathrm{kg}$, following which hemophagocytic syndrome developed, and death ensued on the 61st day (Ogawa et al 2006). Before treatment with tocilizumab, this patient had shown variations in hepatic function and CRP, as well as elevated EBV-DNA levels in plasma. Two weeks after treatment with tocilizumab, hepatic function was increased in association with elevated plasma EBV-DNA, and hemophagocytic syndrome occurred. It was later ascertained that this patient had had Hodgkin's disease before treatment. The other serious adverse events were allergic pneumonitis and infection secondary to a grade 2-3 burn on the leg, and both were cured by medication. Among abnormal laboratory values, increased total cholesterol (TC) was seen in $44 \%$ of patients receiving tocilizumab. However, the increase did not persist, and HDL cholesterol (HDL-C) was similarly elevated. Long-term evaluations of safety will be required to assess any change in the risk of ischemic heart disease. Such an increase in TC concentration was also subsequently reported in treatment with a TNF- $\alpha$ inhibitor (Kiortsis et al 2006), and therefore it may be secondary to the 
(\%)

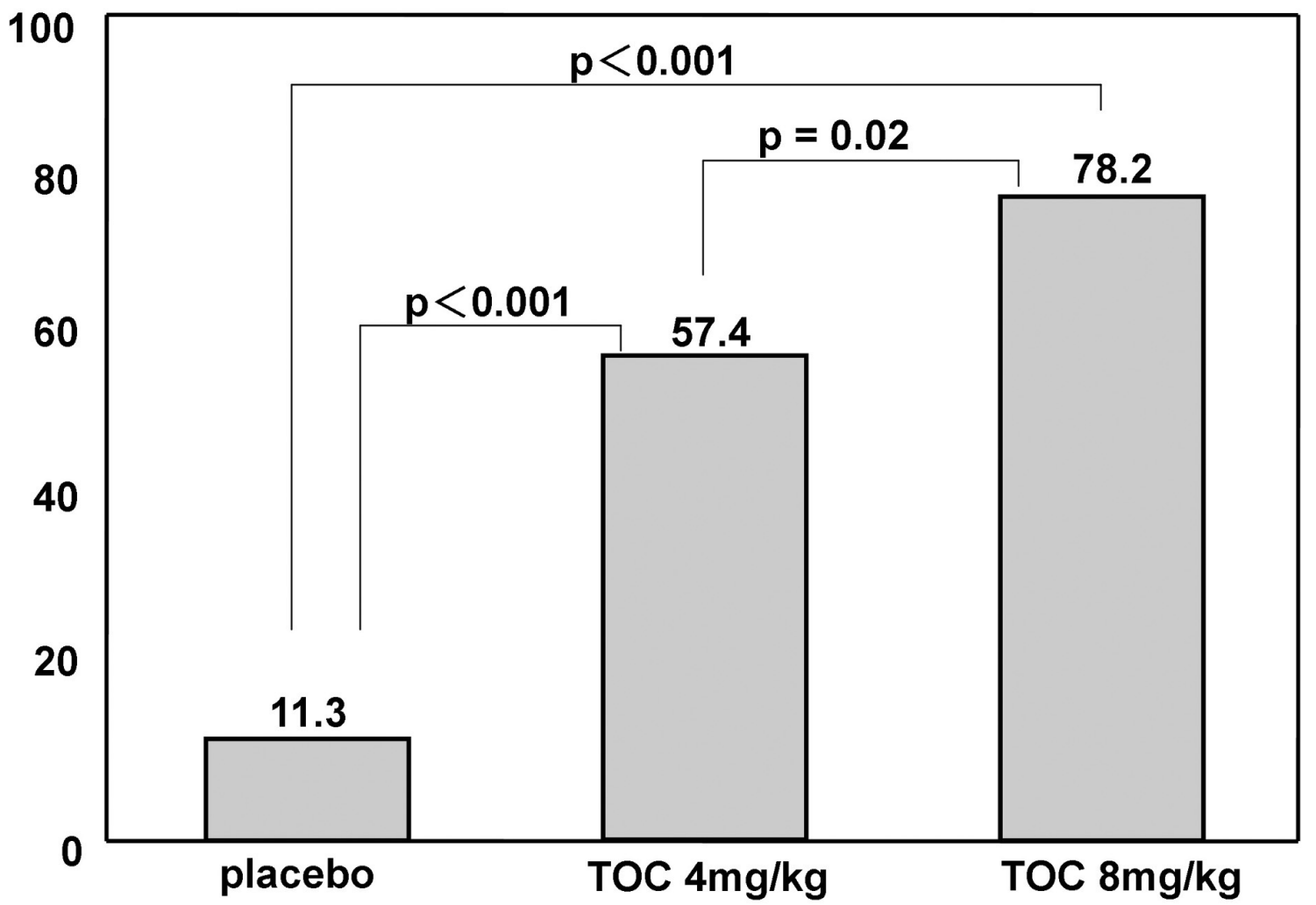

Figure 3 The incidence of a $20 \%$ improvement in disease activity according to the American College of Rheumatology (ACR) criteria at week I2. Tocilizumab treatment significantly improved all measures of disease activity in the ACR core set, and a dose-response relationship was observed between the 4-mg and 8-mg groups. Adapted with permission from Nishimoto N, Yoshizaki K, Miyasaka N, et al. 2004. Treatment of rheumatoid arthritis with humanized anti-interleukin-6 receptor antibody: a multicenter, double-blind, placebo-controlled trial. Arthritis Rheum, 50:I76I-9. Copyright @ 2004.Wiley-Liss, Inc., a subsidiary of John Wiley \& Sons, Inc.

Abbreviation: TOC, tocilizumab.

improvement in inflammation. Liver function disorders and decreases in white blood cell count were also observed in the tocilizumab group, but most were moderate or less severe, and resolved without any treatment. Anti-tocilizumab antibodies were detected in 2 patients treated with tocilizumab, but both were asymptomatic. No induced production of anti-nuclear antibodies (ANA) or anti-DNA antibodies was seen, as is occasionally reported in treatment with TNF- $\alpha$ inhibitors.

\section{Phase II clinical trial (Europe; the Chugai humanized anti-Human recombinant interleukin-6 monoclonal antibody [CHARISMA] study)}

This double-blind, randomized, 16-week trial included 359 patients with RA who had not responded adequately to $\geq 4$ weeks of methotrexate (Maini et al 2006). Patients received 4 infusions of tocilizumab 2, 4 , or $8 \mathrm{mg} / \mathrm{kg}$ every 4 weeks plus either MTX or placebo once weekly, or 4 placebo infusions every 4 weeks plus MTX once weekly. An ACR20 response was achieved by $61 \%$ and $63 \%$ of patients who received 4 and $8 \mathrm{mg} / \mathrm{kg}$ of tocilizumab monotherapy, respectively, and by $63 \%$ and $74 \%$ of patients who received 4 and $8 \mathrm{mg} / \mathrm{kg}$ of tocilizumab plus MTX, respectively. The efficacy of TNF inhibitors is increased by concomitant treatment with MTX, but the authors found that the benefits of tocilizumab were similar with and without concomitant MTX. Tocilizumab was generally well tolerated; about $50 \%$ of patients experienced an adverse event, although most were mild or moderate. Some tocilizumab-treated individuals experienced clinically significant increases in transaminase levels, which seemed to be exacerbated by MTX. Dose-dependent increases in bilirubin and TC, and decreased neutrophil count were also observed. Overall, anti-tocilizumab antibodies developed in 25 patients, all of whom were in the group receiving either $2 \mathrm{mg} / \mathrm{kg}$ or $4 \mathrm{mg} / \mathrm{kg}$ of tocilizumab as monotherapy; anti-tocilizumab antibodies developed in none of the patients receiving treatment with $8 \mathrm{mg} / \mathrm{kg}$ of tocilizumab or combination therapy. 


\section{Phase III clinical trial (Japan)}

Study of active controlled monotherapy used for rheumatoid arthritis, an IL-6 inhibitor (SAMURAI)

This was an X-ray reader-blinded, open-label, randomized, controlled trial in RA patients with disease duration less than 5 years, and inadequate to at least 1 DMARD (Nishimoto et al 2007). Three hundred and six RA patients with inadequate response to DMARDS were randomized to tocilizumab monotherapy (tocilizumab group, 158 patients) or conventional therapy (conventional DMARDs group, 148 patients). Patients in the tocilizumab group received $8 \mathrm{mg} / \mathrm{kg}$ of tocilizumab every 4 weeks, a total of 13 times, while those in the control group received conventional treatment with any conventional DMARDs, except for TNF inhibitors or leflunomide. At week 52, patients in the tocilizumab group showed statistically less radiographic progression, as measured by the change in total modified Sharp score (TSS), than those receiving DMARDs ( $2.3 \pm$ 5.6 versus $6.1 \pm 11.4 ; p=0.001$ ). Tocilizumab was superior to DMARDs in preventing both erosion and joint space narrowing ( $p<0.001$ and $p=0.018$ respectively). This study showed the superiority of blocking IL- 6 with tocilizumab compared with conventional DMARDs in inhibiting radiographic progression.

Study of active controlled tocilizumab monotherapy for rheumatoid arthritis patients with inadequate response to methotrexate (SATORI)

This phase III double-blind controlled trial investigated the efficacy and safety of tocilizumab in monotherapy in patients with active RA who had inadequate responses to MTX treatment (Nishimoto et al 2006a). Patients with active RA despite receiving MTX $8 \mathrm{mg} /$ week, the recommended dose in Japan, during the 8-week screening period, were randomized in a ratio of $1: 1$ to receive either tocilizumab $8 \mathrm{mg} /$ week plus MTX placebo (tocilizumab group) or tocilizumab placebo plus MTX $8 \mathrm{mg} /$ week (MTX group) for 24 weeks. Of 127 patients enrolled, 125 patients received at least one dose of the study drugs (61 patients in the tocilizumab group and 64 patients in the MTX group). At 24 weeks, the ACR20 response rate was statistically significantly higher in the tocilizumab group than in the MTX group $(80.3 \%$ versus $25.0 \%, \mathrm{p}<0.001)$. Patients in the tocilizumab group also had significantly higher ACR50 and ACR70 response rates than patients treated with MTX $(49.2 \%$ versus $10.9 \%$, $\mathrm{p}<0.001$ and $29.5 \%$ versus $6.3 \%, \mathrm{p}<0.001$, respectively). Seven patients and 31 patients were withdrawn from the tocilizumab and MTX groups, respectively. Withdrawal due to adverse events ( tocilizumab $n=2$, MTX $n=3$ ) as well as the occurrence of serious adverse events (tocilizumab $n=4, \operatorname{MTX} n=3$ ) were similar between the groups. Increased lipids were noted in the tocilizumab group but became stable at around the upper limit of normal range. The frequency of liver function test abnormalities as adverse events was higher in the MTX group (ALT increase 10.9\%) but the severity was grade 1 in most cases. No tuberculosis was reported. In this study tocilizumab in monotherapy for 24 weeks significantly improved signs and symptoms and was well tolerated in patients who had active RA despite receiving MTX.

\section{Advantages of tocilizumab and future prospects}

The late phase II and two phase III clinical trials in Japan showed similarly reproducible outstanding results (Nishimoto et al 2006b) (Table 1). From these findings, it can be anticipated that monotherapy with tocilizumab $8 \mathrm{mg} /$ $\mathrm{kg}$ will yield equivalent clinical efficacy to TNF inhibitor in combination with MTX. In the CHARISMA study conducted in Europe, the difference in ACR20 response between the tocilizumab monotherapy $8-\mathrm{mg} / \mathrm{kg}$ group and the MTX plus tocilizumab $8-\mathrm{mg} / \mathrm{kg}$ combination therapy group was not significant. However, the numbers of patients achieving ACR50 and ACR70 responses tended to be higher in the combination therapy group. A detailed evaluation of the clinical efficacy, safety, and inhibitory effect on bone and cartilage destruction of combination therapy with MTX plus tocilizumab will be revealed in the phase III trials that are currently underway in Europe, North America, and elsewhere. Noteworthy aspects of treatment with tocilizumab are that SAA and CRP levels were normalized in nearly all the patients in whom pre-infusion trough levels of the drug can be detected in blood, that is, tocilizumab can be measured in blood before treatment. In the phase III SATORI

Table I DAS28 remission $(<2.6) \%$ improvement scores among tocilizumab's clinical studies. Tocilizumab treatment significantly improved the measures of DAS28 scores compared to the control group. Adapted from Nishimoto et al (2006b)

\begin{tabular}{|c|c|c|c|c|c|c|}
\hline & \multicolumn{2}{|c|}{ Late P II } & \multicolumn{2}{|c|}{ SATORI } & \multicolumn{2}{|c|}{ SAMURAI } \\
\hline & $\mathbf{T}$ & C & $\mathbf{T}$ & C & $\mathbf{T}$ & C \\
\hline 12 weeks & 18.2 & 0 & 34.3 & 1.6 & 29.3 & 1.4 \\
\hline 24 weeks & N.A & N.A & 41.0 & 1.6 & 40.1 & 4.1 \\
\hline 52 weeks & N.A & N.A & N.A & N.A & 58.6 & 3.4 \\
\hline
\end{tabular}

Notes: SATORI = Study of active controlled monotherapy for rheumatoid arthritis patients with inadequate response to methotrexate; SAMURAI = Study of active controlled monotherapy used for rheumatoid arthritis, an IL-6 inhibitor. 
trial conducted in Japan, patients received $8 \mathrm{mg} / \mathrm{kg}$ every 4 weeks for 24 weeks, and the patients who were unable to achieve CRP normalization were those in whom tocilizumab could not be detected in blood at trough levels. Trough levels could not be maintained in $10 \%-20 \%$ of patients, and if it were possible to adjust the dosing interval in these patients so that the trough level could be maintained, it is predicted that the improvement rate in clinical results would increase. As regards prognostic factors of joint destruction in the SAMURAI trial, the percentage change in matrix metalloproteinase-3 (MMP-3) at 12 weeks after starting treatment was an independent prognostic factor for the erosion score at 52 weeks by the modified Sharp method, and the percentage changes in CRP, MMP-3, and serum N-terminal propeptide of type II A collagen at 12 weeks after starting treatment have been reported to be significantly independent prognostic factors for the joint space narrowing (JSN) score (Hashimoto et al 2007). In the SAMURAI trial, the protocol also called for infusion of $8 \mathrm{mg} / \mathrm{kg}$ every 4 weeks, and the fact that CRP was identified as a risk factor for the JSN score can be considered to be a result that suggests the importance of maintaining pre-infusion trough levels for preventing destruction of cartilage and joints. Since tocilizumab is a humanized rather than a chimeric antibody, it is unlikely that anti-tocilizumab antibodies will be produced. In the CHARISMA study, however, in the low-dose groups treated with tocilizumab monotherapy $2 \mathrm{mg} / \mathrm{kg}$ and $4 \mathrm{mg} / \mathrm{kg}$, the concentration of tocilizumab in blood could not be detected at the trough level, and in these groups, anti-tocilizumab antibodies were produced in a small number of patients. In the SAMURAI trial, antitocilizumab antibodies were produced in 4 patients, but the trough blood concentration could not be detected in 3 of these patients (Nishimoto et al 2007), and it is considered that maintaining the pre-infusion trough level is critical to improving the efficacy rate and reducing infusion reactions. These patients were withdrawn in accordance with the study protocol. Furthermore, in patients in whom the treatment was effective once, there was almost no decrease in the efficacy of tocilizumab, in other words the escape phenomenon was absent in the extension study, and this should be a major advantage of tocilizumab. Hence, when using tocilizumab in the post-approval clinical setting, it will be advisable in the early period after infusion to either monitor the blood concentration of tocilizumab or to adjust the dosing interval for individual patients using CRP or SAA as surrogate markers so that the trough level is maintained. Furthermore, since the capacity of tocilizumab to normalize values of CRP and SAA is clearly superior to that of TNF inhibitors (Hagiwara et al 2004, 2005; Okuda et al 2007), the advantage in treatment of AA amyloidosis, a serious complication of rheumatic diseases (Okuda et al 2006) should be emphasized.

However, as well as yielding normalization of CRP and SAA levels, inhibition of IL-6 by tocilizumab makes IL-6induced fever and general fatigue less prominent. Therefore, it is vital to undertake careful clinical observations and monitoring so that discovery after the onset of infection is not delayed and the infection does not become more severe.

Turning now to the effect on tuberculosis seen in treatment with TNF inhibitors, it should be noted that while TNF plays an important role in granuloma formation (Roach et al 2002), IL-6 has no such action. For tuberculosis therefore, it is considered that there will be little likelihood of recrudescence in patients with previous tuberculosis infection, and this is also considered to be an advantage over TNF inhibitors. As regards abnormal variations in laboratory values, increased TC was seen in about $40 \%$ of patients in the tocilizumab treatment group, a finding that was dose-dependent. However, since the increased TC value subsequently leveled off, and HDL-C was also increased, the atherogenic index was unchanged. Increased serum IL-6 levels have been associated with future risk of cardiovascular events (Fisman et al 2006), but long-term epidemiological surveillance will be needed to ascertain whether tocilizumab acts protectively against or promotes cardiovascular events. The emergence of ANA or anti-DNA antibodies occasionally observed with TNF inhibitor was also not evident in either trial in treatment with tocilizumab. Another interesting report notes that when NZB/NZW F1 mice with a spontaneous autoimmune disease that resembles systemic lupus erythematosus (SLE) were administered with anti-mouse IL-6 receptor antibodies, the production of IgG-class anti-DNA antibodies was almost completely inhibited resulting in the inhibition of nephritis, hence the development of nephritis was inhibited, and the survival time prolonged (Mihara et al 1998). Tocilizumab therefore promises to be effective in the treatment of SLE and other autoimmune diseases.

\section{Footnote}

These findings have been published in the primary publications referred to, and listed in the reference list.

\section{Disclosures}

The author has no conflicts of interest to declare. 


\section{References}

Choy EH, Isenberg DA, Garrood T, et al. 2002. Therapeutic benefit of blocking interleukin-6 receptor monoclonal antibody in rheumatoid arthritis: a randomized, double-blind, placebo-controlled, dose-escalation trial. Arthritis Rheum, 46:3143-50.

Fisman EZ, Benderly M, Esper RJ, et al. 2006. Interleukin-6 and the risk of future cardiovascular events in patients with angina pectoris and/or healed myocardial infarction. Am J Cardiol, 98:14-18.

Hagiwara K, Nishikawa T, Isobe T, et al. 2004. IL-6 plays a critical role in synergistic induction of human serum amyloid A (SAA) gene when stimulated with proinflammatory cytokines as analyzed with an SAA isoform real-time quantitative RT-PCR assay system. Biochem Biophys Res Commun, 314:363-9.

Hagiwara K, Nishikawa K, Sugamata Y, et al. 2005. Essential role of STAT3 in cytokine-driven NF- $\mathrm{KB}$-mediated serum amyloid A gene expression. Gene Cells, 10:1051-63.

Hashimoto J, Garnero P, Miyasaka N, et al. 2007. Early changes in biochemical markers of cartilage turnover and synovial inflammation after tocilizumab monotherapy predict the one-year radiographic progression in patients with early rheumatoid arthritis. Mod Rheumatol, 17:S78.

Hirano T, Yasukawa K, Harada H, et al. 1986. Complementary DNA for a novel human interleukin (BSF-2) that induces B lymphocytes to produce immunoglobulin. Nature, 324:73-6.

Kiortsis DN, Mavridis AK, Filippatos TD, et al. 2006. Effects of infliximab treatment on lipoprotein profile in patients with rheumatoid arthritis and ankylosing spondylitis. J Rheumatol, 33:921-3.

Kotake S, Sato K, Kim KJ, et al. 1996. Interleukin-6 and soluble interleukin-6 receptors in the synovial fluids from rheumatoid arthritis patients are responsible for osteoclast-like cell formation. J Bone Miner Res, 11:88-95.

Lubberts E, Koenders MI, Oppers-Walgreen B, et al. 2004. Treatment with a neutralizing anti-murine interleukin-17 antibody after the onset of collagen-induced arthritis reduces joint inflammation, cartilage destruction, and bone erosion. Arthritis Rheum, 50:650-9.

Maini RN, Taylor PC, Szechinski J, et al. 2006. Double-blind randomized controlled clinical trial of the interleukin-6 receptor antagonist, tocilizumab, in European patients with rheumatoid arthritis who had an incomplete response to methotrexate. Arthritis Rheum, 54:2817-29.

Mihara M, Takagi Y, Takeda Y, et al. 1998. IL-6 receptor blockage inhibits the onset of autoimmune kidney disease in NZB/W F1 mice. Clin Exp Immunol, 112:397-402.

Morgan ME, Flierman R, van Duivenvoorde LM, et al. 2005. Effective treatment of collagen-induced arthritis by adoptive transfer of CD25+ regulatory T cells. Arthritis Rheum, 52:2212-21.

Nakahara H, Song J, Sugimoto M, et al. 2003. Anti-interleukin-6 receptor antibody therapy reduces vascular endothelial growth factor (VEGF) production in rheumatoid arthritis. Arthritis Rheum, 48:1521-9.
Nishimoto N, Maeda K, Kuritani T, et al. 2003. Toxicity, pharmacokinetics, and dose finding study of repetitive treatment with humanized antiinterleukin 6 receptor antibody MRA in rheumatoid arthritis. Phase I/II clinical study. J Rheumatol, 30:1426-35.

Nishimoto N, Yoshizaki K, Miyasaka N, et al. 2004. Treatment of rheumatoid arthritis with humanized anti-interleukin-6 receptor antibody: a multicenter, double-blind, placebo-controlled trial. Arthritis Rheum, 50:1761-9.

Nishimoto N, Miyasaka N, Yamamoto K, et al. 2006a. Efficacy and safety of tocilizumab in monotherapy, an anti-IL-6 receptor monoclonal antibody, in patients with active rheumatoid arthritis: results from a 24 week double-blind phase III study. Ann Rheum Dis, 65 (Suppl II):59.

Nishimoto N, Miyasaka N, Yamamoto K, et al. 2006b. Consistent clinical response resulted from three Japanese randomized controlled trials of tocilizumab, humanized anti-IL-6 receptor antibody, in active rheumatoid arthritis (RA) patients. Arthritis Rheum, 54:S410.

Nishimoto N, Hashimoto J, Miyasaka N, et al. 2007. Study of active controlled monotherapy used for rheumatoid arthritis, an IL-6 inhibitor (SAMURAI): evidence of clinical and radiographic benefit from an $\mathrm{X}$-ray reader-blinded randomized controlled trial of tocilizumab. Ann Rheum Dis, 66:1162-7.

Ogawa J, Harigai M, Akashi T, et al. 2006. Exacerbation of chronic active Epstein-Barr virus infection in a patient with rheumatoid arthritis receiving humanised anti-interleukin-6 receptor monoclonal antibody. Ann Rheum Dis, 65:1667-9.

Okuda Y, Takasugi K. 2006. Successful use of a humanized anti-interleukin-6 antibody, tocilizumab, to treat amyloid A amyloidosis complicating juvenile idiopathic arthritis. Arthritis Rheum, 54:2997-3000.

Okuda Y, Ohnishi M, Matoba K, et al. 2007. Clinical evaluation of anticytokine therapies for AA amyloidosis complicating rheumatoid arthritis-in view of suppression of SAA levels. Mod Rheumatol, 17:S81.

Roach DR, Bean AG, Demangel C, et al. 2002. TNF regulates chemokine induction essential for cell recruitment, granuloma formation, and clearance of mycobacterial infection. J Immunol, 168:4620-7.

Roux-Lombard P, Eberhardt K, Saxne T, et al. 2001. Cytokines, metalloproteinases, their inhibitors and cartilage oligometric matrix protein: relationship to radiological progression and inflammation in early rheumatoid arthritis. A prospective 5-year study. Rheumatology, 40:544-51.

Ward LD, Howlett GJ, Discolo G, et al. 1994. High affinity interleukin-6 receptor is a hexameric complex consisting of two molecules each of interleukin-6, interleukin-6 receptor, and gp-130. J Biol Chem, 269:23286-9.

Yoshizaki K, Nakagawa T, Kaieda T, et al. 1982. Induction of proliferation and Ig production in human B leukemic cells by anti-immunoglobulins and T cell factors. J Immunol, 128:1296-301. 